\title{
Intrachromosomal Amplification of Chromosome 21 in Childhood Acute Lymphoblastic Leukemia: Study of 3 Cases
}

\author{
Aleksandra Mroczkowskaa, ${ }^{a} \quad$ Monika Lejman $^{c}$ \\ aLaboratory of Genetic Diagnostics, University Children's Hospital, Lublin, Poland; \\ ${ }^{b}$ Department of Hematology, Blood Neoplasms and Bone Marrow Transplantation, \\ Laboratory of Molecular Biology and Cytogenetics, Wroclaw Medical University, Wroclaw, \\ Poland; ' Laboratory of Genetic Diagnostics, Medical University of Lublin, Lublin, Poland
}

\section{Keywords}

Intrachromosomal amplification of chromosome 21 . Acute lymphoblastic leukemia .

Fluorescence in situ hybridization · Single-nucleotide polymorphismarray

\begin{abstract}
Acute lymphoblastic leukemia (ALL) is the most common malignancy of childhood. The presence or absence of a characteristic genetic abnormality usually observed in childhood ALL plays a very important role in determining the prognosis and stratification for treatment. Intrachromosomal amplification of chromosome 21 (iAMP21) is an uncommon high-risk chromosomal abnormality than can occur only in $2 \%$ of childhood B-cell precursor lymphoblastic leukemia. Molecular genetic analysis and the fluorescence in situ hybridization (FISH) technique are the basic methods used to detect the presence of the most common genetic abnormalities, the presence or absence of which has an impact on the patient's classification into the appropriate risk group. This work presents 3 BCP-ALL iAMP21-positive patients who were detected during routine genetic diagnostics using the FISH method and microarray test.

iAMP21 is associated with a poor prognosis and high risk for relapse. Children with B-cell precursor lymphoblastic leukemia with this genetic entity are associated with a delayed treatment response. The FISH method and single-nucleotide polymorphism array provides a useful method to detect characteristic genetic changes.


Mroczkowska and Lejman: iAMP21 in Childhood ALL

\section{Introduction}

The most common childhood malignancy is acute lymphoblastic leukemia (ALL). About $80-85 \%$ of cases are B-cell precursor ALL (BCP-ALL), while 15-20\% are T-cell leukemias. Diagnostic tests of bone marrow blast cells allow the analysis of characteristic genetic abnormalities, usually determining prognosis and stratification for treatment in childhood ALL [1, 2]. Intrachromosomal amplification of chromosome 21 (iAMP21) is associated with a poor outcome and high relapse risk when the patients were treated by a standard therapy. Detection of iAMP21 is very significant to the choice of appropriate treatment [3-6]. Potentially, iAMP21 will be detected during routine tests by fluorescence in situ hybridization (FISH) if ETV6/RUNX1 probes are used. The interphase nuclei show extra signals from the RUNX1 probe, but metaphases show that signals are located on an abnormal chromosome 21. Some laboratories in routine diagnostics use the break-apart probe to detect only ETV6 gene rearrangement. An important point is that the iAMP21 can be detected by the FISH method exclusively using the ETV6/RUNX1 translocation probe or by performing a microarray test [2, 7-9]. We present 3 cases of BCP-ALL with amplification of RUNX1 detected by FISH and microarray.

\section{Case Presentation}

\section{Case One}

A 14-year-old girl was admitted to the Department of Pediatric Hematology, Oncology, and Transplantology, Medical University of Lublin in Poland due to an anemia and weakness. Lymphadenopathy, hepatosplenomegaly, and the infiltration of the central nervous system were not found. The laboratory results were as follows: white blood cell (WBC) 1,950/ $\mu \mathrm{L}$, platelet count $120,000 / \mu \mathrm{L}$, and hemoglobin level $10.8 \mathrm{~g} / \mathrm{dL}$. Evaluation of the bone marrow showed 73\% blast cells. The girl was diagnosed with preB common positive ALL in May 2018 and she was treated according to the ALL Intercontinental-Berlin-Frankfurt-Münster 2009 (ALL IC-BFM 2009) protocol for the intermediate risk group.

Cytogenetic analysis was performed on a bone marrow sample. G-banded chromosome analysis revealed a female karyotype $47, \mathrm{XX},+$ mar/46,XX. Poor quality of the metaphase chromosomes made them impossible to evaluate. FISH was performed with use of the commercially available probes: BCR/ABL1, KMT2A, ETV6/RUNX1 (Vysis, Abbott Molecular, Des Plaines, IL, USA). No rearrangements were found in $B C R / A B L 1, K M T 2 A$, or ETV6/RUNX1 tests. FISH analysis revealed between 6 and 9 signals of $R U N X 1$ in $80.6 \%$ of the analyzed nuclei. Metaphases showed that extra signals were clustered together and located on abnormal chromosomes difficult to identify (Fig. 1A). CytoScan HD array (Thermo Fisher Scientific, Waltham, MA, USA) was performed on the same sample of bone marrow. This method confirmed iAMP21 $\operatorname{arr}[$ GRCh37] 21q21.3q22.3(28054448_43559902)x4. The size of amplification was $15.5 \mathrm{Mb}$ and contained the following genes: DYRK1A, TMPRSS2, OLIG2, RUNX1, ADAMTS1, TIAM1, ETS2, and ERG. Apart from this, analysis revealed duplication of a fragment of the long arm of chromosome 1, deletion within 4-7 exons of the IKZF1 gene, deletion within chromosome 13 including the RB1 gene (20-27 exon biallelic deletion), and deletion of band 21q22.3 (PTTG1IP, S100B, TFF3, U2AF1, TMPRSS3, TFF1, TFF2, CSTB). Loss of heterozygosity of the entire chromosome 22 was also demonstrated (Fig. 1B). FISH analysis revealed that additional signals were located on a large, hard to identify chromosome. Microarray analysis suggested translocation between chromosome 21 and a fragment of the long arm of chromosome 1. Performing a FISH test with whole chromosome painting probes for chromosomes 1 and 21 (Cytocell Ltd, Oxford Gene Technology, Cambridge, UK) confirmed translocation. Additional RUNX1 signals were clustered together on this translocated chromosome.

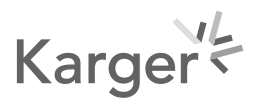


Fig. 1. Case 1. A FISH analysis on metaphase and interphase with an LSI ETV6/RUNX1 ES Dual Color Translocation Probe Set (Vysis). B SNP array revealed the highest level of amplification located within the RUNX1 locus.

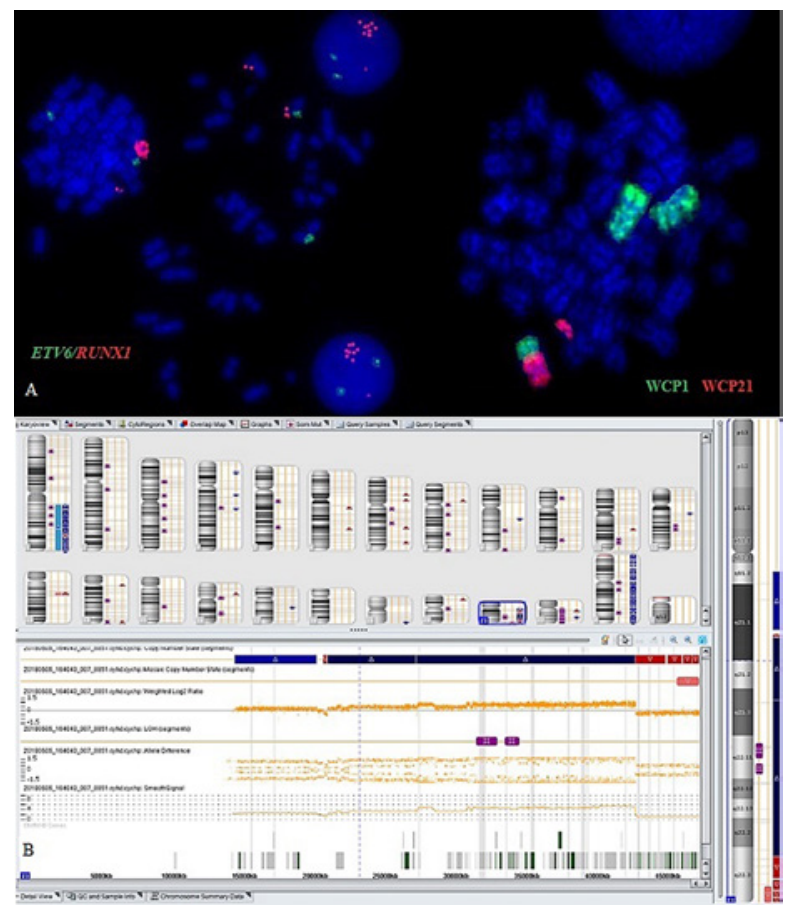

\section{Case Two}

The second patient was a 13-year-old boy who was admitted to the hospital because of recurrent infections and petechiae. Lymphadenopathy, hepatosplenomegaly, and the infiltration of the central nervous system were not observed. A diagnostic test revealed: WBC $1,800 / \mu \mathrm{L}$, platelet count $21,000 / \mu \mathrm{L}$, hemoglobin level $4.1 \mathrm{~g} / \mathrm{dL}$, and $90 \%$ leukemic cells in the bone marrow. The boy was diagnosed with BCP-ALL in August 2017. He presented a good treatment response to steroids, but was FMC-MRD-positive on day 15. Therefore, he was classified as high-risk group. According to the ALL IC-BFM 2009 protocol cytogenetic analysis and FISH were performed on a bone marrow sample. GTG band staining revealed an abnormal male karyotype with a marker chromosome probably derived from chromosome 21 . The FISH study showed no rearrangements in BCR/ABL1, KMT2A, or ETV6/RUNX1. However, FISH revealed 8-9 signals of RUNX1 in 91\% interphase cells (Fig. 2A). CytoScan HD array revealed amplification of $\operatorname{arr}[\mathrm{GRCh} 37]$ 21q11.2q21.1(15006457_22491098)x4, including NRIP1 and MIR125B2 genes and arr[GRCh37] 21q21.3q22.3(28054448_44101079)x4 containing 12 genes: TIAM1, OLIG2, RUNX1, DYRK1A, ERG, ETS2, TMPRSS2, TFF3, ADAMTS1, TFF1, TFF2, and TMPRSS3. The size of amplification was $16.5 \mathrm{Mb}$. In addition, the test also showed deletion of band 21q22.3 (PTTG1IP, S100B, CSTB, U2AF1), loss of heterozygosity within the long arm of chromosome 12 , and a mosaic loss of heterozygosity within the long arm of chromosome 1 (Fig. 2B). The patient qualified for matched unrelated donor bone marrow transplantation.

\section{Case Three}

The third patient was a 14-year-old boy who was admitted to hospital due to a fever that had lasted for 5 days, and pain of the lower limbs. Lymphadenopathy, hepatosplenomegaly, and the infiltration of the central nervous system were not observed, but the WBC count was $3,900 / \mu \mathrm{L}$, platelet count was $359,000 / \mu \mathrm{L}$, and hemoglobin level was $9.5 \mathrm{~g} / \mathrm{dL}$. Bone marrow evaluation showed $69 \%$ blasts. The boy was diagnosed with B-cell precursor common positive 


\section{Case Reports in Oncology}

\begin{tabular}{l|l}
\hline Case Rep Oncol 2021;14:592-598 \\
\hline DOI: 10.1159/000514107 & $\begin{array}{l}\text { @ 2021 The Author(s). Published by S. Karger AG, Basel } \\
\text { www.karger.com/cro }\end{array}$ \\
\hline
\end{tabular}

Mroczkowska and Lejman: iAMP21 in Childhood ALL

Fig. 2. Case 2. A FISH analysis on metaphase and interphase with an LSI ETV6/RUNX1 ES Dual Color Translocation Probe Set (Vysis). B SNP array revealed the highest level of amplification located within the RUNX1 locus.

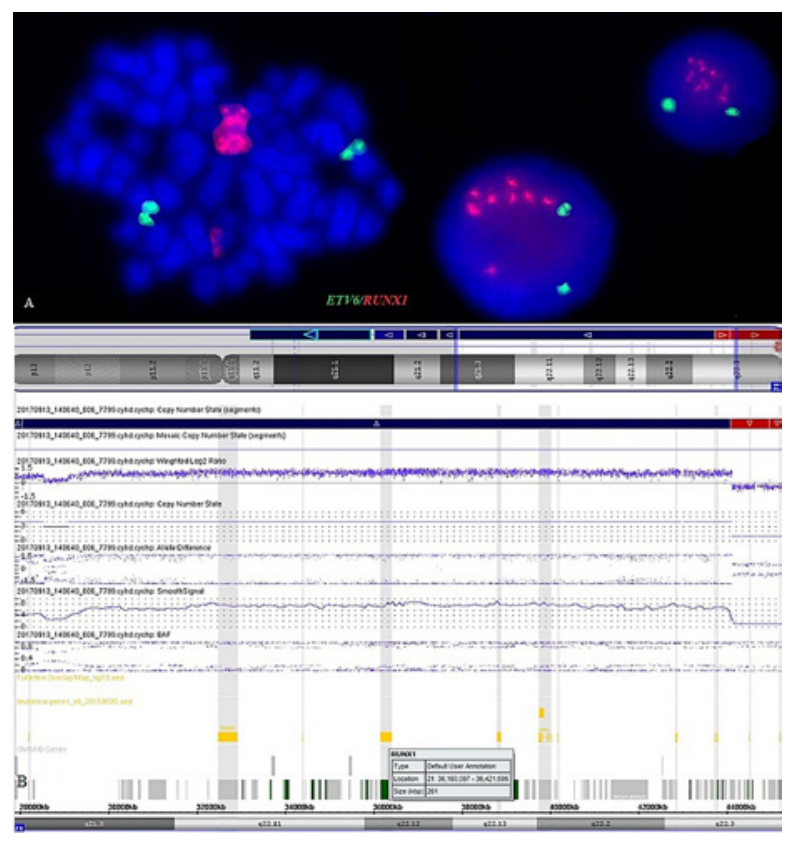

Fig. 3. Case 3. A FISH analysis on metaphase and interphase with an LSI ETV6/RUNX1 ES Dual Color Translocation Probe Set (Vysis). B SNP array revealed the highest level of amplification located within the RUNX1 locus.

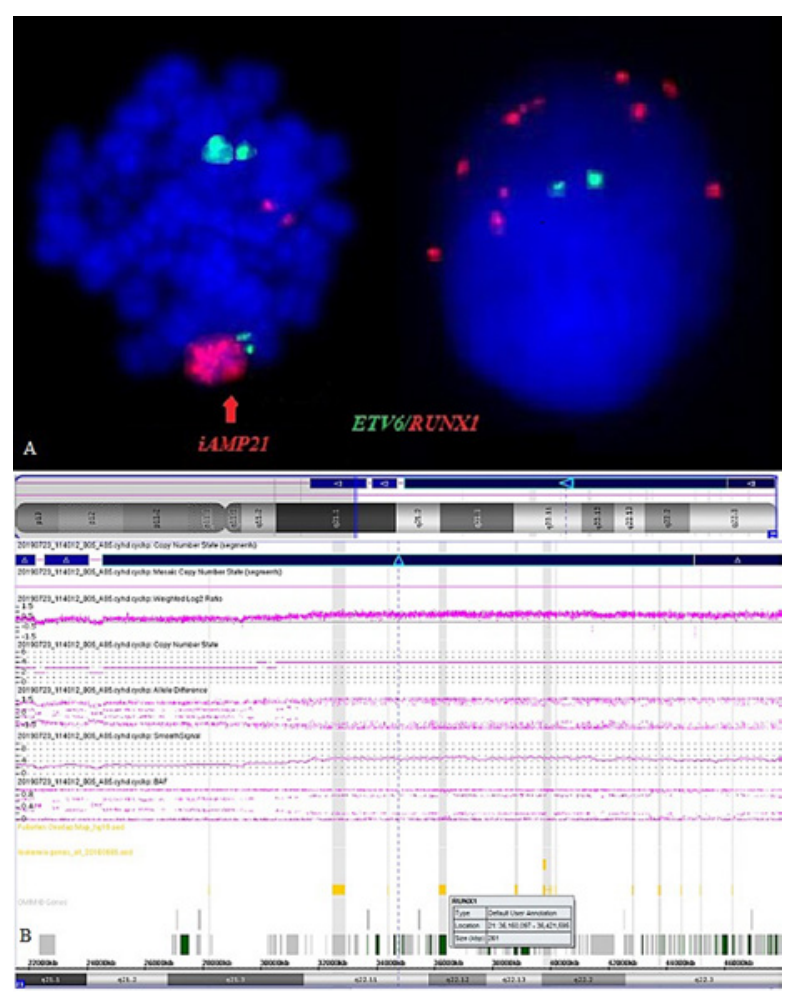

ALL and chemotherapy was started in July 2019, according to the AIEOP-BFM ALL 2017 protocol. He was classified into the early non-high-risk group. According to the protocol cytogenetic analysis and FISH were performed on a bone marrow sample. G-banded chromosome analysis revealed a male karyotype $45, \mathrm{XY},-21,+$ mar $/ 46, \mathrm{XY}$. The FISH study revealed no rearrangements in BCR/ABL1, KMT2A, or ETV6/RUNX1, but revealed 8-10 signals of RUNX1 in $24.4 \%$ of interphase cells (Fig. 3A). CytoScan HD array revealed amplification of arr[GRCh37] 21q22.11q22.3(31846769_47960084)x4. The size of amplification was $16.1 \mathrm{Mb}$ and included 
following genes: OLIG2, TIAM1, ERG, ETS2, TMPRSS3, TFF2, TFF3, TFF1, DYRK1A, U2AF1, RUNX1, TMPRSS2, CSTB, and PTTG1IP. Furthermore, analysis showed duplication of the entire long arm of chromosome 9 and deletion within chromosome 14q23.2-q31.3 (Fig. 3B).

\section{Discussion}

iAMP21 defines a distinct cytogenetic subgroup of childhood BCP-ALL which was identified in 2003 [8]. It was detected by performing FISH for the presence of the ETV6/RUNX1 fusion. This entity is a rare chromosomal abnormality that can occur in approximately $2 \%$ of pediatric patients with BCP-ALL. Patients with BCP-ALL and iAMP21 are characterized by older age and a low WBC count, similar to our presented patients $[3,5,7,8,10]$. Moorman et al. [5] observed that the presence of this abnormality is associated with an increased risk of relapse. Overall survival and event-free survival are inferior in patients with iAMP21, as was observed by Heerema et al. [4]. Our patients are in a stable and continuous state of remission, but the observation time is too short. The 5.1-Mb region, which included the RUNX1 gene, miR-802, and genes mapping to the Down syndrome critical region, is the most common region of amplification on chromosome $21[3,10]$. In our cases, CytoScan HD array revealed size of amplification from 15.5 to $16.5 \mathrm{Mb}$. In addition, some patients present a deletion at the telomeric end of chromosome $21[2,10]$. Microarray analysis revealed a deletion of chromosome 21 at the telomeric end in 2 of our 3 patients.

The most common method to identify this entity is FISH. iAMP21 is defined as a presence of three or more extra copies of RUNX1 on a structurally abnormal chromosome 21 or a total of five or more RUNX1 signals per cell. The RUNX1 gene is used as a marker, although there is no evidence that this gene is the target of this abnormality $[2,11]$. Recent studies have shown that the most common genetic abnormality among patients with iAMP21 is chromosomal instability of chromosome 21 , which suggests that it is a primary genetic event $[3,10]$.

$R U N X 1$ is a transcription factor which plays a very important role in regulating the development of hematopoiesis and hematopoietic stem cell homeostasis in mammals. RUNX1 regulates the expression of the hematopoiesis-specific genes, including cytokines such as IL-3 and GM-CSF, cytokine receptors, for example M-CSFR, T and B receptors, and megakaryocytespecific genes such as PF4. Furthermore, RUNX1 is the most frequently mutated gene of hematological malignancies, such as acute myeloid leukemia, ALL, chronic myelomonocytic leukemia, and myelodysplastic syndrome. In childhood ALL, the most common chromosome abnormality is $\mathrm{t}(12 ; 21)$, which is present among $17 \%$ of young patients. The fusion is generated by $\mathrm{t}(12 ; 21)$ between ETV6 on chromosome 12 and RUNX1 on chromosome 21 (ETV6/RUNX1). This abnormality leads to loss or impairment RUNX1 function $[10,11]$.

In routine diagnostics, detection of iAMP21 is possible only with a probe that detects ETV6/RUNX1 translocations. In this way we can detect one of the most common changes $\mathrm{t}(12$; 21) and one of the rare changes: iAMP21. Conventional G-banding karyotype and the FISH technique is a standard method for detecting the most common structural abnormalities observed in BCP-ALL (BCR/ABL1, KMT2A, ETV6/RUNX1, TCF3). However, cytogenetic analysis sometimes has a diagnostic problem because of cell culture failure or poor quality of GTG staining. FISH analysis is mostly aberration specific and requires a large number of unique probes [12]. For this reason, our laboratory performs molecular karyotype tests as a standard for patients with BCP-ALL. Single-nucleotide polymorphism (SNP) array is a powerful tool to complete a conventional cytogenetic (CC) analysis and FISH method. This method is able to detect numerical and structural chromosomal rearrangements, gains and deletions of genomic regions (copy number variations), and copy-neutral loss-of-heterozygosity which cannot be detected by CC and FISH. Chromosomal microarray analysis enables detection of chromo-

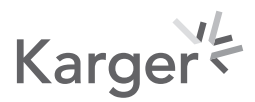


somal aberrations at the exon level [12-14]. This method helps identify recurrent genetic abnormalities occurring in ALL: IKZF1, CDKN2A/B, PAX5, ETV6, ERG, and RB1 [15]. According to the new AIEOP-BFM ALL 2017 protocol the SNP microarray is recommended for determining the status of the IKZF ${ }^{\text {plus }}$ group in children with BCP-ALL $[12,14]$. The poor quality of metaphase chromosomes in our patients made them difficult to evaluate. Genetic changes were identified by molecular karyotyping.

Analysis of SNP microarray revealed that among our patients they all had ERG gene amplification, one additionally had deletion in the IKZF1 and RB1 genes. Furthermore, for our first patient SNP microarray helped to determine the origin of the marker chromosome.

iAMP21 is an uncommon abnormality which can be identified in the routine process of searching for ETV6/RUNX1 fusion. IAMP21 has been associated with a poor prognosis and this genetic entity is important for classification into the appropriate risk group. Using the SNP microarray method, we observed other abnormalities which cannot be observed in cytogenetic analysis, the presence of which may be relevant to patient stratification into the relevant risk group.

\section{Statement of Ethics}

This study was approved by the ethics committee of the Medical University of Lublin, Poland (reference No. KE-0254/222/2012). Written informed consent was obtained from the parents of the patients for publication of the case report and any accompanying images.

\section{Conflict of Interest Statement}

The authors have no conflicts of interest to declare.

\section{Funding Sources}

No funding was obtained for this study.

\section{Author Contributions}

M.L. was responsible for the conception and design of the study. M.L. and A.M. conducted the laboratory work. M.L. and A.M. were responsible for the interpretation of data. A.M. prepared the final manuscript for publication. All authors read and approved the final manuscript.

\section{References}

1 Tasian SK, Loh ML, Hunger SP. Childhood acute lymphoblastic leukemia: integrating genomics into therapy. Cancer. 2015;121(20):3577-90.

2 Moorman A. The clinical relevance of chromosomal and genomic abnormalities in B-cell precursor acute lymphoblastic leukaemia. Blood Rev. 2012;26(3):123-35.

3 Harrison C, Moorman A, Schwab C, Carroll A, Raetz E, Devidas M, et al. International Workshop in Childhood Acute Lymphoblastic Leukemia. An international study of intrachromosomal amplification of chromosome 21 (iAMP21): cytogenetic characterization and outcome. Leukemia. 2014;28(5):1015-21. 
4 Heerema NA, Carroll AJ, Devidas M, Loh ML, Borowitz MJ, Gastier-Foster JM, et al. Intrachromosomal amplification of chromosome 21 is associated with inferior outcomes in children with acute lymphoblastic leukemia treated in contemporary standard-risk children's oncology group studies: a report from the Children's Oncology Group. J Clin Oncol. 2013;31(27):3397-402.

5 Moorman AV, Richards SM, Robinson HM, Strefford JC, Gibson BE, Kinsey SE, et al. Prognosis of children with acute lymphoblastic leukemia (ALL) and intrachromosomal amplification of chromosome 21 (iAMP21). Blood. 2007;109(6):2327-30.

6 Soulier J, Trakhtenbrot L, Najfeld V, Lipton JM, Mathew S, Avet-Loiseau H, et al. Amplification of band q22 of chromosome 21, including AML1, in older children with acute lymphoblastic leukemia: an emerging molecular cytogenetic subgroup. Leukemia. 2003;17(8):1679-82.

7 Johnson RC, Weinberg OK, Cascio MJ, Dahl GV, Mitton BA, Silverman LB, et al. Cytogenetic variation of B-lymphoblastic leukemia with intrachromosomal amplification of chromosome 21 (iAMP21): a multi-institutional series review. Am J Clin Pathol. 2015;144(1):103-12.

8 Rand V, Parker H, Russell LJ, Schwab C, Ensor H, Irving J, et al. Genomic characterization implicates iAMP21 as a likely primary genetic event in childhood B-cell precursor acute lymphoblastic leukemia. Blood. 2011; 117(25):6848-55.

9 Harrison C. Blood spotlight on iAMP21 acute lymphoblastic leukemia (ALL), a high-risk pediatric disease. Blood. 2015;125(9):1383-6.

10 Sood R, Kamikubo Y, Liu P. Role of RUNX1 in hematological malignancies. Blood. 2017;129(15):2070-82.

11 Bonifer C, Levantini E, Kouskoff V, Lacaud G. Runx1 structure and function in blood cell development. Adv Exp Med Biol. 2017;962:65-81.

12 Berry NK, Scott RJ, Rowlings P, Enjeti AK. Clinical use of SNP-microarrays for the detection of genome-wide changes in haematological malignancies. Crit Rev Oncol Hematol. 2019;142:58-67.

13 Mitrakos A, Kattamis A, Katsibardi K, Papadhimitriou S, Kitsiou-Tzeli S, Kanavakis E, et al. High resolution chromosomal microarray analysis (CMA) enhances the genetic profile of pediatric B-cell acute lymphoblastic leukemia patients. Leuk Res. 2019;83:106177.

14 Berry NK, Scott RJ, Sutton R, Law T, Trahair TN, Dalla-Pozza L, et al. Enrichment of atypical hyperdiploidy and IKZF1 deletions detected by SNP-microarray in high-risk Australian AIEOP-BFM B-cell acute lymphoblastic leukaemia cohort. Cancer Genet. 2020;242:8-14.

15 Chen C, Heng EYH, Lim AST, Lau LC, Lim TH, Wong GC, et al. Chromosomal microarray analysis is superior in identifying cryptic aberrations in patients with acute lymphoblastic leukemia at diagnosis/relapse as a single assay. Int J Lab Hematol. 2019;41(4):561-71. 